\title{
Efficacy And Safety Of 5\% Lidocaine Patches For Postoperative Pain Management In Patients Undergoing Unilateral Inguinal Hernia Repair: Study Protocol For A Prospective, Double-Blind, Randomized, Controlled Clinical Trial
}

Hong-min Ahn

Seoul National University Bundang Hospital

Heung-Kwon Oh ( $\nabla$ crsohk@gmail.com )

Seoul National University Bundang Hospital https://orcid.org/0000-0002-8066-2367

Duck-Woo Kim

Seoul National University Bundang Hospital

\section{Sung-Bum Kang}

Seoul National University Bundang Hospital

\section{Bon-Wook Koo}

Seoul National University Bundang Hospital

\section{Pyung-Bok Lee}

Seoul National University Bundang Hospital

Study protocol

Keywords: Topical analgesics, Lidocaine patch, Inguinal hernia repair

Posted Date: June 28th, 2021

DOl: https://doi.org/10.21203/rs.3.rs-527208/v1

License: (c) (i) This work is licensed under a Creative Commons Attribution 4.0 International License.

Read Full License 
1 Efficacy and safety of $\mathbf{5 \%}$ lidocaine patches for postoperative pain management in 2 patients undergoing unilateral inguinal hernia repair: study protocol for a prospective, 3 double-blind, randomized, controlled clinical trial

5 Hong-min $\mathrm{Ahn}^{1}$, Heung-Kwon $\mathrm{Oh}^{1}$, Duck-Woo Kim ${ }^{1}$, Sung-Bum Kang ${ }^{1}$, Bon-Wook Koo ${ }^{2}$, 6 Pyung-Bok Lee ${ }^{2}$

7

$8 \quad{ }^{1}$ Department of Surgery, Seoul National University Bundang Hospital, Seongnam, Korea

$9 \quad{ }^{2}$ Department of Anesthesiology and Pain Medicine, Seoul National University Bundang 10 Hospital, Seongnam, Korea

Authors' email addresses

markahn85@gmail.com

Corresponding author: Heung-Kwon Oh, MD, PhD

166 Gumi-ro, Bundang-gu, 13620 Seongnam, Republic of Korea

E-mail: crsohk@gmail.com

Corresponding author: Pyung-Bok Lee, MD, PhD 
21166 Gumi-ro, Bundang-gu, 13620, Seongnam, Republic of Korea

22 Tel: $+82-31-787-7495$

23 E-mail: painfree@snubh.org

24

25 Heung-Kwon Oh and Pyung-Bok Lee agree to share the corresponding authorship on this 26 article.

27 


\section{Abstract}

Background: Acute postoperative pain is a common complication of inguinal hernia repair. Pain management using local application of anesthetic agents over the skin surrounding the surgical incision may reduce the requirement for other pain medications. Targeted topical analgesics such as 5\% lidocaine patches have been known to improve acute and chronic pain. However, the clinical effect of lidocaine patches on postoperative pain after inguinal hernia repair has not been studied, especially in patients undergoing surgery at day surgery units.

Methods/design: This is a single-center, prospective, double-blind, randomized, controlled clinical trial. Participants with unilateral inguinal hernia will be randomized to the lidocaine patch group or the placebo patch group. Based on the randomized allocation sequence, either lidocaine patches or placebo patches will be attached near each participant's surgical wound after open hernia repair under general anesthesia. Participants will be asked to follow-up at our outpatient clinic on the first postoperative day and at one week after surgery. The primary outcome is pain intensity, which will be measured using the Visual Analogue Scale (VAS) at the time of discharge from the day surgery unit. The secondary outcomes are VAS score at 24 hours and one week after surgery. We will collect and analyze the participants' clinical data (amount of intraoperative opioid use, time to recovery, and pain intensity at 30 min after surgery) and demographic characteristics (age, sex, body weight, and height).

Discussion: This trial may not only provide evidence on the efficacy of a 5\% lidocaine patch for acute postoperative pain management after unilateral inguinal hernia repair, but also demonstrate the efficacy and safety of the patch for post-discharge pain management.

Trial registration: ClinicalTrials.gov: NCT04754451 (registered on February 10, 2021). https://clinicaltrials.gov/ct2/show/NCT04754451 
51 Keywords: Topical analgesics, Lidocaine patch, Inguinal hernia repair 
Inguinal hernia repair is a commonly performed in general surgery. Acute postoperative pain, which can be moderate to severe in intensity, commonly occurs after open inguinal hernia repair $(1,2)$. The intensity of pain after open inguinal hernia repair has been reported to be the highest on the first postoperative day (1). Untreated postoperative pain or a high pain score within 24 hours of surgery may lead to persistent pain after inguinal hernia repair $(3,4)$. Patients with prolonged pain have an increased duration of hospitalization and may fail to return to their daily lives (5). For these reasons, various modalities, such as pain medication and nerve block, have been used for postoperative pain management $(3,6)$.

Acute postoperative pain is primarily managed with intravenous or oral administration of narcotic or non-narcotic analgesics. However, most of these drugs are systemically administered, which may result in complications such as nausea, vomiting, inhibition of bowel motility, and abdominal discomfort. These adverse effects can occur even after discharge from the hospital, especially in patients undergoing day surgery for inguinal hernia repair.

On the other hand, pain management with topical applied analgesics near the surgical incision site may reduce the incidence of complications and drug interactions (7). A lidocaine patch is a pain management modality that involves local infiltration of an analgesic. The use of a $5 \%$ lidocaine patch has only been approved by the Food and Drug Administration for treating neurologic pain due to Herpes zoster infection; it has not been approved for acute postoperative pain management (8).

Several studies have demonstrated the positive effects of lidocaine patches on acute postoperative pain $(9,10)$. However, to the best of our knowledge, there are no studies on the clinical effects of lidocaine patches for acute postoperative pain management after inguinal 
75 hernia repair, especially in patients admitted to day surgery units. Furthermore, the use of a 76 lidocaine patch may minimize the systemic effects of the analgesic agent and reduce the 77 financial burden on the patient by decreasing the requirement for the extra pain medication. 78 Therefore, we designed this double-blind, randomized, controlled clinical trial to determine the 79 efficacy and safety of a $5 \%$ lidocaine patch for postoperative pain management in patients 80 undergoing unilateral inguinal hernia repair at a day surgery unit. 
This is a single-center, prospective, double-blind, randomized, controlled clinical trial. This study has been approved by the Institutional Review Board (B-2007-625-006) of Seoul National University Bundang Hospital. Written informed consent to participate will be obtained from all the participants. After surgery, the experimental group will receive 5\% lidocaine patches (Lidotop Cataplasma, SK chemical Co. Ltd, Seongnam, Korea), and the control group will receive placebo patches that were specially made for this study. The overview of the study design is shown in Figure 1.

Inclusion and exclusion criteria

93 The inclusion criteria are: (a) age between 19 and 80 years, (b) American Society of 94 Anesthesiologists risk classification score of I (healthy) or II (mild systemic disease), (c) 95 elective open surgery for unilateral inguinal hernia, and (d) admission to and discharge from 96 the day surgery unit on the day of the surgery itself.

97 The exclusion criteria are: (a) obesity (body mass index $>30 \mathrm{~kg} / \mathrm{m}^{2}$ ), (b) renal insufficiency 98 (serum creatinine level $\geq 1.4 \mathrm{mg} / \mathrm{dL}$ ), (c) liver insufficiency (serum aspartate transferase level $99 \geq 120 \mathrm{IU} / \mathrm{L}$ or serum alanine transaminase level $\geq 120 \mathrm{IU} / \mathrm{L}$ ), (d) known hypersensitivity to 100 amide-based local anesthetics, (e) use of class I antiarrhythmic medication such as tocainide and mexiletine, and (f) refusal to participate. 
104 The primary outcome is pain intensity, which will be measured using the Visual Analogue Scale 105 (VAS) at the time of discharge from the day surgery unit. We will evaluate each patient's VAS scores in three situations: at rest, when coughing, and when moving. The secondary outcomes are pain intensity at 24 hours and one week after surgery. We will evaluate each patient's VAS score when they visit the clinic for wound dressing on the first postoperative day. At the followup visit one week after surgery, we will assess not only the VAS score but also the rates of postoperative complications, such as nausea, vomiting, and desaturation, and the total amount demographic characteristics, such as age, sex, body weight, and height, will also be recorded.

Sample size calculation

\section{Randomization}


patch group. We will achieve block randomization using Random Allocation Software (Isfahan University of Medical Sciences, Isfahan, Iran). A research nurse from the anesthesiology department who is not involved in the study will generate the randomized allocation sequence and prepare the patches. The patches will be given to the anesthesiologist and surgeon responsible for applying it at the end of the surgery. Thus, the researchers who evaluate the patients' clinical parameters will be blinded to the group allocation.

\section{Procedure}

All the participants will undergo unilateral inguinal hernia repair under general anesthesia. As pre-treatment, each patient will be injected with $0.03 \mathrm{mg} / \mathrm{kg}$ of midazolam on arrival at the operating room. An anesthesiologist will induce and maintain general anesthesia with inhaled anesthetics and opiates using conventional methods. A general surgeon will make a transverse incision of approximately 3 to $4 \mathrm{~cm}$ along the skin fold line and perform routine Lichtenstein tension-free mesh inguinal hernia repair. The skin will be closed with subcutaneous interrupted sutures, and skin bond ( $7 \mathrm{~mm} \times 60 \mathrm{~mm}$; Leukosan SkinLink, BSN medical, Hamburg, Germany) will be applied. After covering the surgical wound with aseptic dressing, lidocaine or placebo patches will be applied near the surgical wound based on the randomized allocation sequence. Each patient will receive two patches, which will be placed $1 \mathrm{~cm}$ above and $1 \mathrm{~cm}$ below the surgical wound (Fig. 2).

We will allow participants to use additional pain medications such as opioids or nonsteroidal anti-inflammatory drugs as needed. Either fentanyl ( $50 \mu \mathrm{g}$; Hana fentanyl citrate, Hana Pharm. Co., Ltd, Seoul, Korea) or ketorolac (30 mg; Trolac, Whan In Pharm. Co., Ltd, Seoul, Korea) will be prescribed. Fentanyl will be prescribed a maximum of three times when a patient 
experiences pain with a VAS score of $>5$. Ketorolac will be prescribed if a patient experiences any adverse effect following fentanyl administration. Oral medications such as tramadol/acetaminophen (37mg/325 mg; Rapicet, Chong Kun Dang Pharm. Co., Seoul, Korea) will be prescribed to each patient at the time of discharge for pain management.

Data analysis

154 All statistical analyses will be performed using Statistical Package for the Social Sciences 155 version 25.0 for Windows (SPSS/IBM, Chicago, IL, USA). The participants' demographic characteristics will be analyzed using descriptive statistics. After verifying the normality, the Student t-test or Mann-Whitney $U$ test will be used to analyze the numerical data. For categorical data, we will use the Chi-squared test or Fisher's exact test. All results with a pvalue of less than 0.05 will be considered as significant. 


\section{Discussion}

162 Targeted topical analgesics such as 5\% lidocaine patches have been known to improve acute and chronic pain $(7,11)$. Previous studies have investigated the effects of lidocaine patches on postoperative pain management following various surgeries. Most of these studies included patients with small trocar wounds after laparoscopic surgeries, such as laparoscopic appendectomy, ventral hernia repair, and gynecologic surgery. A prospective study of 40 patients who underwent laparoscopic appendectomy in whom a lidocaine patch was applied at the umbilical trocar site showed that a 5\% lidocaine patch might be effective for managing port-site pain (12). Another study of 30 patients who underwent laparoscopic ventral hernia repair also found that lidocaine patches are effective for postoperative pain management (9). A study of 40 patients who underwent laparoscopic gynecologic surgery found that patients who received a lidocaine patch had lower VAS scores and additional analgesic requirement than those who did not receive a lidocaine patch (10).

There are a few studies in which a lidocaine patch was used postoperatively for incisions that were longer than a trocar incision. In a randomized controlled trial of 70 patients who underwent radical retropubic prostatectomy with a lower midline incision, a lidocaine patch (14).

Through these studies, which included randomized clinical trials and meta-analyses, the safety and efficacy of using a 5\% lidocaine patch for acute postoperative pain management has been established. However, most of these studies were on patients who received hospital care and 
patient-controlled analgesia following surgery, in which the patients' acute pain was rapidly treated by medical staff.

We have designed this clinical trial to investigate the efficacy of a $5 \%$ lidocaine patch for postoperative pain management without additional medical attention. Therefore, we will enroll patients undergoing open unilateral inguinal hernia repair at a day surgery unit. We believe that in addition to demonstrating the efficacy of a $5 \%$ lidocaine patch on acute postoperative pain after unilateral inguinal hernia repair, we will be able to provide evidence on its efficacy and safety for post-discharge pain management. In summary, we hypothesize that a 5\% lidocaine patch is an effective option for acute postoperative pain management following unilateral inguinal hernia repair, and it can be safely used in an outpatient clinical setting.

\section{Trial status}

Patient recruitment commenced in March 2021, and the predicted date of completion of this study is October 2021.

\section{List of abbreviations}


205 This study has been approved by the Institutional Review Board (B-2007-625-006) of Seoul

206 National University Bundang Hospital. Written informed consent to participate will be 207 obtained from all the participants.

208

209 Consent for publication

210 Not applicable

212 Availability of data and materials

213 Not applicable

214

215 Competing interests

216 The authors declare that they have no competing interests

217

$218 \quad$ Funding

219 This trial is supported by SK chemical Co. Ltd

220

221

Authors'contributions 
222 PBL is the chief investigator; he conceived the study and supervised the proposal and protocol 223 development. HKO and BWK contributed to the study design and proposal development. 224 DWK and SBK were the lead trial methodologist. HA and HKO organized the protocol and 225 wrote the manuscript. All authors read and approved the final manuscript.

Acknowledgements

228 The authors appreciate the assistance provided by the team of surgery and anesthesiology at 229 Seoul National University Bundang Hospital for help during this trial.

Authors' information

232

${ }^{1}$ Department of Surgery, Seoul National University Bundang Hospital, Seongnam, Korea

233

${ }^{2}$ Department of Anesthesiology and Pain Medicine, Seoul National University Bundang 234 Hospital, Seongnam, Korea

235

236 


\section{References}

238

239

240

241

242

243

244

245

246

247

248

249

250

251

252

253

254

255

256

257

258

259

1. Callesen T, Bech K, Nielsen R, Andersen J, Hesselfeldt P, Roikjaer O, et al. Pain after groin hernia repair. British Journal of surgery. 1998;85(10):1412-4.

2. Joshi GP. Multimodal analgesia techniques and postoperative rehabilitation. Anesthesiol Clin North Am. 2005;23(1):185-202.

3. Joshi GP, Rawal N, Kehlet H, collaboration P, Bonnet F, Camu F, et al. Evidence-based management of postoperative pain in adults undergoing open inguinal hernia surgery. Br J Surg. 2012;99(2):168-85.

4. Karaman Y, Ozkarakas H, Karaman S, Turan M, Gonullu M, Uyar M, et al. [Incidence of chronic pain after ingunial hernia repair]. Agri. 2015;27(2):97-103.

5. Aasvang EK, Gmaehle E, Hansen JB, Gmaehle B, Forman JL, Schwarz J, et al. Predictive risk factors for persistent postherniotomy pain. The Journal of the American Society of Anesthesiologists. 2010;112(4):957-69.

6. Yazicioglu D, Caparlar C, Akkaya T, Mercan U, Kulacoglu H. Tizanidine for the management of acute postoperative pain after inguinal hernia repair: A placebo-controlled double-blind trial. Eur J Anaesthesiol. 2016;33(3):215-22.

7. Argoff CE. Targeted topical peripheral analgesics in the management of pain. Current pain and headache reports. 2003;7(1):34-8.

8. Gammaitoni AR, Alvarez NA, Galer BS. Safety and tolerability of the lidocaine patch 5\%, a targeted peripheral analgesic: a review of the literature. J Clin Pharmacol. 2003;43(2):111-7.

9. Saber AA, Elgamal MH, Rao AJ, Itawi EA, Martinez RL. Early experience with lidocaine patch for postoperative pain control after laparoscopic ventral hernia repair. Int J Surg. 2009;7(1):36-8. 
260 10. Kwon YS, Kim JB, Jung HJ, Koo YJ, Lee IH, Im KT, et al. Treatment for postoperative 261 wound pain in gynecologic laparoscopic surgery: topical lidocaine patches. J Laparoendosc 262 Adv Surg Tech A. 2012;22(7):668-73.

263 11. Bai Y, Miller T, Tan M, Law LS, Gan TJ. Lidocaine patch for acute pain management: a 264 meta-analysis of prospective controlled trials. Curr Med Res Opin. 2015;31(3):575-81.

265 12. Lee W, Hahn K, Hur J, Kim Y. Effect of Topical Lidocaine Patch on Postoperative Pain 266 Management in Laparoscopic Appendectomy: A Randomized, Double-Blind, Prospective 267 Study. J Laparoendosc Adv Surg Tech A. 2018;28(9):1061-7.

268 13. Habib AS, Polascik TJ, Weizer AZ, White WD, Moul JW, ElGasim MA, et al. Lidocaine 269 patch for postoperative analgesia after radical retropubic prostatectomy. Anesth Analg. $270 \quad 2009 ; 108(6): 1950-3$.

271 14. Lau LL, Li CY, Lee A, Chan SK. The use of 5\% lidocaine medicated plaster for acute 272 postoperative pain after gynecological surgery: A pilot randomized controlled feasibility trial. 273 Medicine (Baltimore). 2018;97(39):e12582. 
Figure legends

277

278 Figure 1. Trial design flowchart. VAS, Visual Analogue Scale; POD, Postoperative day

279

280 Figure 2. Location of the patch. Irrespective of the type of patch used (5\% lidocaine or 281 placebo), one patch will be applied $1 \mathrm{~cm}$ above and another $1 \mathrm{~cm}$ below the surgical wound.

282 
Figures

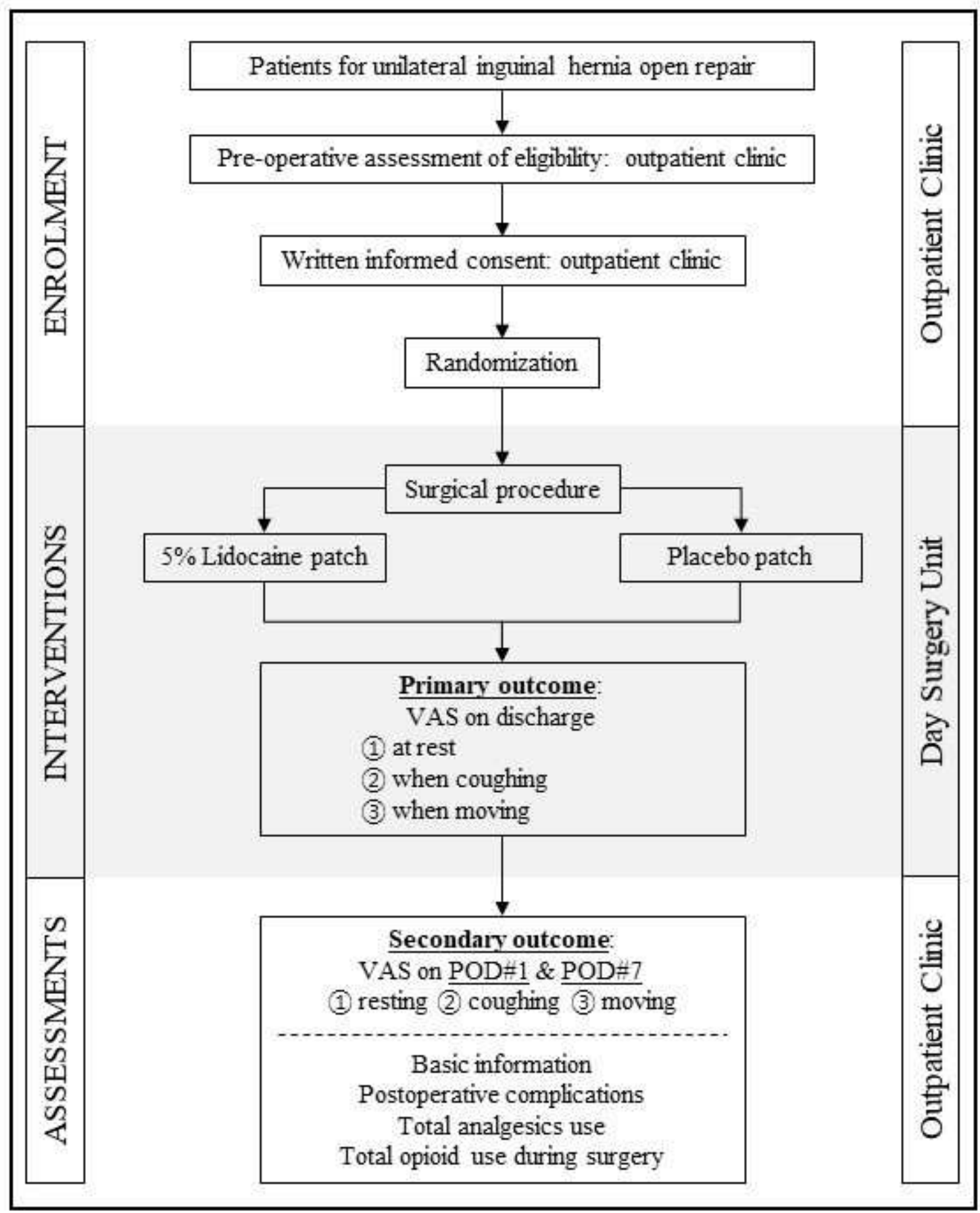

Figure 1

Trial design flowchart. VAS, Visual Analogue Scale; POD, Postoperative day 


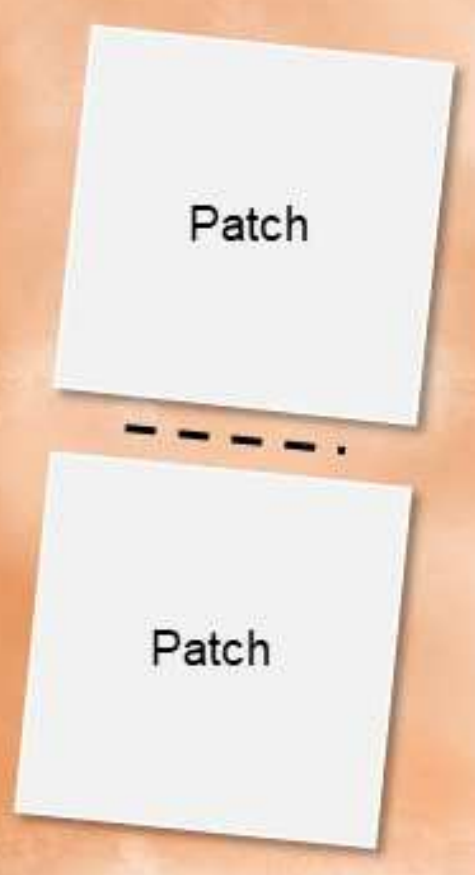

Figure 2

Location of the patch. Irrespective of the type of patch used (5\% lidocaine or placebo), one patch will be applied $1 \mathrm{~cm}$ above and another $1 \mathrm{~cm}$ below the surgical wound.

\section{Supplementary Files}

This is a list of supplementary files associated with this preprint. Click to download.

- SPIRITchecklistLidoPatch.docx 